\title{
Miscibility effect of biodegradable aliphatic poly(butylene succinate)/aromatic polycarbonate blends
}

\author{
Thandi Patricia Gumede ${ }^{1 *}$, Katekani Shingange ${ }^{2}$, Pontsho Mbule ${ }^{3}$, Bennie Motloung ${ }^{4}$
}

${ }^{1}$ Department of Life Sciences, Central University of Technology, Free State, Bloemfontein, South Africa

${ }^{2}$ Centre for Nanostructures and Advanced Materials (CeNAM), DSI-CSIR Nanotechnology Innovation Centre, Council for Scientific and Industrial Research, Pretoria 0001, South Africa ${ }^{3}$ Department of Physics, College of Science, Engineering and Technology, University of South Africa, Johannesburg, 1710, South Africa

${ }^{4}$ Department of Chemistry and Polymer Science, Stellenbosch University, Private Bag X1, Matieland 7602, South Africa

\begin{abstract}
.
Biodegradable polymers are gaining attention for applications in drug delivery, tissue engineering, and wound management. Methods for enhancing their potential in these applications include blending non-biodegradable polymers with biodegradable polymers to form blends with unique properties. Herein we report on the effect of non-biodegradable PC on the physical, mechanical, and thermal properties of biodegradable PBS. The PBS/PC blends contained 3, 13, and 27 wt.\% PC and were prepared by melt extrusion. The FTIR results revealed apparent compatibility between the two polymers. Even though the blends are compatible, the extent of miscibility depends on how well PC is dispersed in the PBS matrix. According to the SEM micrographs, adding 3 wt.\% PC resulted in a miscible polymer blend. However, above this content, agglomeration was observed suggesting possible phase segregation. XRD results revealed peak shifts to higher angles and new peaks forming between $25-30^{\circ}$. The shift could be related to the interaction between the components in the blends. The crystallinity was also improved at $3 \mathrm{wt}$.\% PC and this is consistent with the DSC results. TGA analysis indicated no improvement in the thermal stability of the blends. DMA revealed that at low PC content (i.e., 3 wt.\%), there is a marked improvement in the elastic modulus.
\end{abstract}

\section{*Corresponding author: tgumede@ cut.ac.za}


Keywords: blends, compatibility, miscibility, nucleation, reinforcement

\section{Introduction}

Plastic waste is a growing concern worldwide. The governments and many organizations are working to save the environment by utilizing biodegradable materials. Biodegradable polymers are materials that can fully decompose into carbon dioxide, methane, water, biomass and inorganic compounds under aerobic or anaerobic conditions. They should reduce waste and address the problem of a shortage in landfill availability [1-4].

Biodegradable polymers consist of a family of polyesters and they have demonstrated massive growth towards sustainability. The ester groups in polyesters can undergo hydrolytic chain scissions which has made biodegradable polyesters to be at the forefront of polymer science both in fundamental and application research [5]. Polyesters can be categorized into two major groups: aliphatic (linear) polyesters, and aromatic (ring) polyesters. Aliphatic polyesters include poly( $\varepsilon$-caprolactone) (PCL), poly(butylene succinate) (PBS), polylactide (PLA), poly(hydroxyalkanoates) (PHA), poly(hydroxybutyrate) (PHB), and poly(hydroxybutyrate-co-hydroxyvalerate) (PHBV), while aromatic polyesters include bisphenol A polycarbonate (PC), polyethylene terephthalate (PET), poly(propylene terephthalate) (PPT), poly(butylene terephthalate) (PBT) and poly(butylene adipate-coterephthalate) (PBAT) [6-8]. Among the polyesters, aliphatic polyesters received the most attention as they are widely employed in tissue engineering and other bio-medical applications such as drug delivery, medical implants, scaffolds, etc. $[9,10]$. They have adjustable degradation rate, excellent processability, good biocompatibility and reproducibility. However, some of their applications are limited due to the polymers' poor thermal and mechanical properties such as brittleness, low toughness, and slow crystallization rates [11].

In order to improve the properties of some aliphatic polyesters such PCL, which has a relatively low mechanical strength and still maintain its biodegradability; several authors have blended it with other aliphatic polyesters such as PBS, PLA, and PHBV [12-20]. Among the blends, blending PCL with PBS was found to be interesting because of the mutual improvement in the properties of the individual polymers [11-17]. Generally, the authors reported that the tensile strength decreased as the PCL content increased in the blends, while the elongation at break and impact strength increased with increasing PCL content. The decreased tensile strength indicated poor interfacial adhesion between the components in the blends because of the softening effect of PCL. 
Although the field of biodegradable polymers has previously been limited to aliphatic polyesters and/or aliphatic polyester blends; introducing aromatic rings to the polymer structure give rise to much broader thermal, mechanical and biodegradation properties. Aliphatic/aromatic polyester blends show outstanding thermal and mechanical properties due to the presence of the aromatic ring structures while aliphatic esters ensure that the biodegradability of the polymer is maintained [5]. Biodegradable aliphatic/aromatic polyester blends contain a large portion of the aliphatic ester groups because of their high electrophilicity as compared to the aromatic derivatives. Therefore, the presence of aromatic rings within the chain structure decreases the biodegradability of the resulting material.

Several aliphatic/aromatic polyester blends have been investigated in the literature [2130]. Mohapatra et al. [30] studied the morphology, thermal and mechanical properties of biodegradable PLA/PBAT/layered silicate blend nanocomposites. Generally, the tensile strength, percentage elongation and impact strength of the PLA/PBAT blend nanocomposites increased as compared to the neat PLA matrix. The DSC and TGA results also showed an improvement in the thermal properties in comparison to the neat PLA. In other studies [21-27], the blending of biodegradable PCL with a non-biodegradable PC was found to be more interesting because only a single glass transition temperature for the PCL component was reported with its position determined by the composition of the blend, an indication of a miscible polymer blend with improved mechanical performance.

In contrast, some authors [28,29] blended PC/MWCNTs masterbatch with either biodegradable PCL or with biodegradable PBS and evidenced partial miscibility between PCrich and PCL-rich phases in one case, and between PC-rich and PBS-rich phases in another case. However, the extent of miscibility was different for each system and the establishment of a PC-rich phase and the PCL-rich and PBS-rich phases. For both systems, in the PC-rich phase, the small amount of PCL or PBS chains included within this phase plasticized the PC component and the PC-rich phase was therefore able to crystallize. However, in the PCL-rich phase, the presence of the PC chains caused changes in the glass transition temperature of the PCL phase that were much smaller than those predicted by the Fox theory, while in the PBSrich phase, the amount of the PC chains present gave rise to increases in the glass transition temperature of the PBS phase. Therefore, the interfacial tension, polar component surface energy and isothermal crystallization results indicated that the MWCNTs disperse better into the PBS-rich phase than into the PCL-rich phase.

Several research studies based on the effect of non-biodegradable PC/MWCNTs masterbatch on the properties of biodegradable PCL and biodegradable PBS, as well as the 
effect of non-biodegradable PC on the properties of biodegradable PCL have been widely investigated. However, very little information is available that deals with the effect of nonbiodegradable PC on the physical, mechanical, and thermal properties of biodegradable PBS for a variety of applications in the engineering and biomedical fields. Thus, this contribution aims to investigate, analyze, and compare the results collected with the ones available in the literature.

\section{Experimental}

\subsection{Materials}

A commercial poly(1,4-butylene succinate) (PBS), extended with 1,6-diisocyanatohexane, was purchased from Sigma-Aldrich (Johannesburg, South Africa). It has a density of $1.3 \mathrm{~g} \mathrm{~cm}^{-3}$ at $25^{\circ} \mathrm{C}$ and a melting temperature of $120^{\circ} \mathrm{C}$. The weight-average molecular weight $\left(M_{w}\right)$ of PBS is $63,000 \mathrm{~g} \mathrm{~mol}^{-1}$ [26]. It has a melt flow index (MFI) of $20.9 \mathrm{~g} .10 \mathrm{~min}^{-1}\left(190{ }^{\circ} \mathrm{C} / 2.16 \mathrm{~kg}\right)$.

A commercial grade bisphenol-A polycarbonate (Makrolon ${ }^{\circledR} 2407$ ) produced and supplied in pellet form by Bayer Material Science, Germany and having a melt flow index of $20 \mathrm{~g} .10 \mathrm{~min}^{-1}\left(300{ }^{\circ} \mathrm{C} / 1.2 \mathrm{~kg}\right)$ and $\mathrm{M}_{\mathrm{w}}=26576 \mathrm{~g} \mathrm{~mol}^{-1}$ was used.

The blends were prepared through melt extrusion, which was operated under nitrogen atmosphere $\left(50 \mathrm{rpm}, 170{ }^{\circ} \mathrm{C}, 10 \mathrm{~min}\right)$. The calculated weight percentages of the different components in each of the investigated blends are given in Table 1.

Table 1 Weight percentages of the components in the blends.

\begin{tabular}{|c|c|}
\hline PBS (\%) & PC (\%) \\
\hline 100 & 0 \\
\hline 97 & 3 \\
\hline 87 & 13 \\
\hline 73 & 27 \\
\hline 0 & 100 \\
\hline
\end{tabular}

\subsection{Sample Characterization}

To study the chemical structures of the samples, attenuated total reflectance Fourier-transform infrared spectroscopy using a Platinum-ATR Bruner Alpha II was performed. The samples were analysed from the wavelength range of $400-4000 \mathrm{~cm}^{-1}$ at a resolution of $4 \mathrm{~cm}^{-1}$. 
The surface morphology of the samples was examined using a ZEIS-AURIGA scanning electron microscopy (SEM) at an accelerating voltage of $3.0 \mathrm{kV}$.

To examine the crystal phase and purity of the samples, a computer controlled Panalytical X'pert PRO PW3040/60 X-ray diffractometer with a $\mathrm{Cu}-\mathrm{K} \alpha(\lambda=1.5405 \AA)$ radiation was employed.

Thermal degradation studies of the samples were performed using a PerkinElmer Pyris 1 TGA Q500 instrument. The samples with a mass of approximately $10 \mathrm{mg}$ were heated from 25 to $600{ }^{\circ} \mathrm{C}$ at a heating rate of $10{ }^{\circ} \mathrm{C} \mathrm{min}-1$ under nitrogen atmosphere (flow rate of $20 \mathrm{~mL}$ $\left.\min ^{-1}\right)$.

Thermal transitions that occur in the prepared samples were analyzed using a DSC Q2000 instrument from TA instruments, New Castle, DE, USA. The samples with a mass of approximately $5 \mathrm{mg}$ were heated from -20 to $200{ }^{\circ} \mathrm{C}$ at a heating rate of $10{ }^{\circ} \mathrm{C} \mathrm{min}{ }^{-1}$, and then maintained at that temperature for $5 \mathrm{~min}$. The samples were then cooled to $-20{ }^{\circ} \mathrm{C}$ at a cooling rate of $10{ }^{\circ} \mathrm{C} \mathrm{min}-1$ and kept constant for $5 \mathrm{~min}$, then heated to $200{ }^{\circ} \mathrm{C}$ at a heating rate of $10{ }^{\circ} \mathrm{C}$ $\min ^{-1}$. The heating and cooling cycles were conducted under nitrogen as the purge gas, with a flow rate of $25 \mathrm{~mL} \mathrm{~min}{ }^{-1}$ for all the samples.

Thermo-mechanical properties of the extruded samples were conducted using a Perkin Elmer DMA (Model 8000, Brandford, CT, USA) analyzer in a dual cantilever bending mode. The temperature was measured at a frequency of $1 \mathrm{~Hz}$, strain amplitude of $0.01 \%$ and heating rate of $2{ }^{\circ} \mathrm{C} \mathrm{min}^{-1}$ in the temperature range of -60 to $100{ }^{\circ} \mathrm{C}$ for neat PBS and the PBS/PC blends (to avoid the melting of the PBS phase) and -60 to $190{ }^{\circ} \mathrm{C}$ for neat PC.

\section{Results and Discussion}

\subsection{Chemical structure analysis: Fourier-transform infrared (FTIR) spectroscopy}

The FTIR spectra of neat PBS, neat PC, as well as the PBS/PC blends are shown in Figure 1. Neat PBS is characterized by absorption peaks at $3000 \mathrm{~cm}^{-1}$ (C-H from $\mathrm{CH}_{3}$ groups), $1750 \mathrm{~cm}^{-}$ ${ }^{1}$ ( $\mathrm{C}=\mathrm{O}$ stretching vibration), 1155 and $1045 \mathrm{~cm}^{-1}$ (C-O stretching vibrations) [31]. Neat $\mathrm{PC}$ is characterized by $\mathrm{C}-\mathrm{H}$ aromatic ring vibrations around $3000 \mathrm{~cm}^{-1}, \mathrm{C}=\mathrm{O}$ carbonate group vibrations near $1775 \mathrm{~cm}^{-1}, \mathrm{C}=\mathrm{C}$ vibrations at $1506 \mathrm{~cm}^{-1}$, asymmetric $\mathrm{O}-\mathrm{C}-\mathrm{O}$ carbonate group vibrations in the range $1232-1164 \mathrm{~cm}^{-1}, \mathrm{CH}_{3}$-vibrations at $1081 \mathrm{~cm}^{-1}$, and symmetric $\mathrm{O}-\mathrm{C}-\mathrm{O}$ carbonate group vibrations near $1015 \mathrm{~cm}^{-1}$ [32]. The blending of the two polymers shows characteristic bands/vibrations of both PBS and PC, indicating the successful fabrication of the 
blends. However, the spectra of the PBS/PC blends are dominated by the PBS vibrations. This is probably due to a higher content of this component compared to PC in the blends. The position of the bands in the blends remain unchanged with respect to PBS. However, there is an overlapping of the $\mathrm{C}-\mathrm{H}$ and carbonyl groups, as well as the development of double peaks around $1300 \mathrm{~cm}^{-1}$, suggesting some chemical interaction between the two polymers. The PC peaks around 1506 and $1081 \mathrm{~cm}^{-1}$ as well as the PBS peak at $730 \mathrm{~cm}^{-1}$ are absent in the blends possibly due to apparent compatibility between PBS and PC. For a compatible polymer blend, it is envisaged that the FTIR spectra of the blends should differ from that of the individual polymers due to chemical interactions that result in changes in band intensities, shifts, and broadening [33].

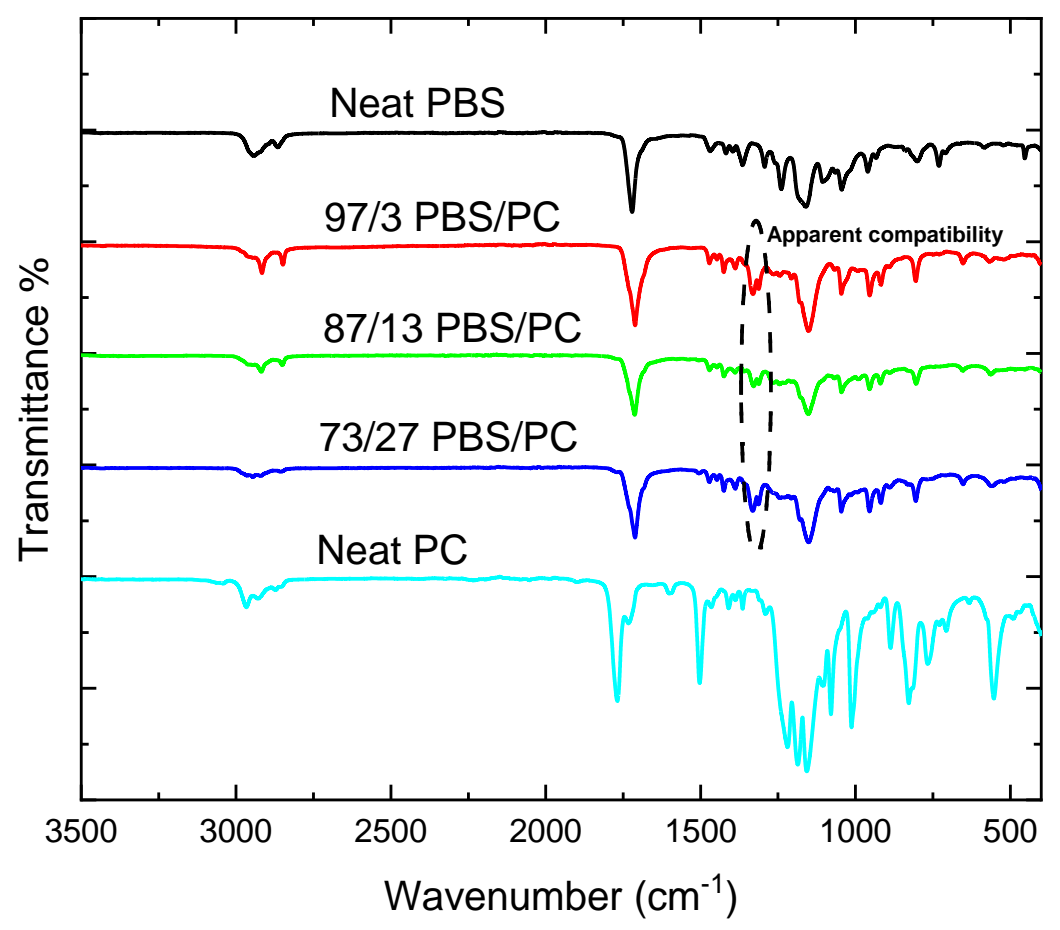

Figure 1 FTIR spectra of neat polymers and the PBS/PC blends.

\subsection{Surface morphology: Scanning electron microscopy analysis (SEM)}

To obtain information about the phase morphology of PBS/PC blends, scanning electron microscopy analyses were performed on various blend ratios and the results are presented in Figure 2. Neat PBS shows a rough surface (Figure 2a) while neat PC appears fairly smooth (Figure $2 b$ ). A rough surface can enhance the adhesion by influencing the adsorption and 
providing larger area for adhesion compared to a smooth surface [34]. The morphology of the resulting blends is shown in Figure 2c-e. At low PC content (e.g., 3 wt.\%), the PBS and PC phases cannot be easily separated from one another, suggesting a miscible polymer blend. However, as the content of PC increases, agglomerates start to form (Figure 2d), and get larger at high PC content (Figure 2e). This indicates poor interaction between the component phases. A similar observation was reported by Luyt et al [29], whereby the size of the dispersed phase (which corresponded to the PC-rich phase) was unexpectedly large considering the amount of the PC that was added in the PBS/PC blends (i.e., 23 wt.\%) indicating a macro-phase segregation in the blends. Since both polymers have similar chemical structures, the two polymers appear to be compatible in all the blend ratios. However, the extent of miscibility depends on the content of the PC added to the blends. In this case, the optimum interfacial adhesion is observed at $3 \mathrm{wt} . \% \mathrm{PC}$, where the two components appear to be miscible. This is not evident above $3 \mathrm{wt} . \%$, where phase-segregation is obvious. 

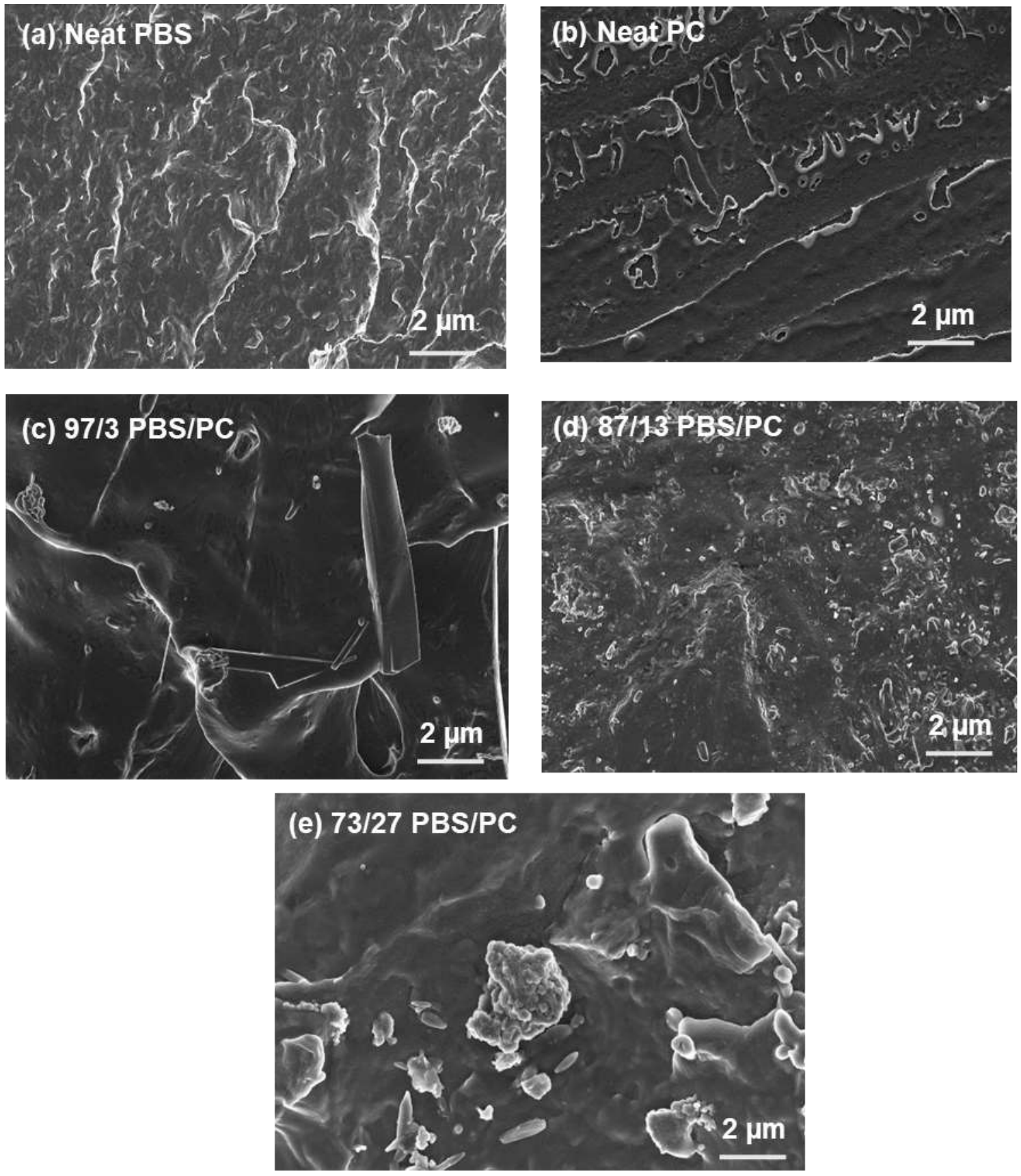

Figure 2 SEM micrographs for (a) neat PBS, (b) neat PC, (c) 97/3 PBS/PC, (d) 87/13 PBS/PC, and (e) 73/27 PBS/PC.

\subsection{Structural properties: $X$-ray diffraction analysis $(X R D)$}

Figure 3 shows the X-ray patterns for neat PBS, neat PC, and the PBS/PC blends. The diffraction peaks of PBS are located at $2 \theta$ values of $19,22,29$ and $44^{\circ}$ which correspond to the (002), (110), (121) and (111) planes, respectively. The diffraction peaks are consistent with the reported monoclinic unit cell of $\alpha$-PBS with unit cell parameters $\mathrm{a}=5.232, \mathrm{~b}=9.057$ and $\mathrm{c}=$ 
$10.900 \AA$ and $\gamma=123.87 \circ$ [29]. The main reflections shown by neat PBS are visible in the Xray patterns of all the blends, while the PBS and PC reflection peaks at $44^{\circ}$ are only visible at low PC content (3 wt.\%). Above this content, this reflection peak is not visible. This is an indication that PC had no effect on the PBS crystalline structure in all the blends except for the 97/3 PBS/PC blend which appeared to be miscible as compared to the other blends and has the highest crystallinity index (see section 3.4).

In addition to the PBS unit cell peaks, there is a peak at $35^{\circ}$ which appears in the blends and becomes less pronounced as the PC content in the blends increase. This peak maybe attributed to the PC component that is partially miscible with the PBS. The same effect was reported by Gumede et al. [29] where such an effect was an indication of partial miscibility between the PBS and PC. Further, the diffraction peaks of the blends are more intense than neat PBS, with the 97/3 PBS/PC blend displaying highly intense diffraction peaks than the rest of the samples, indicating high crystallinity in comparison to the other samples. The high crystallinity of the blends could be attributed to miscibility and nucleation which may have promoted crystallization, therefore resulting in higher crystallinity. A shift in the diffraction peaks to higher 2theta is also observed. Upon increasing the content of PC, there is a peak shift. These peak shifts can be attributed to the phase segregation as reported in the SEM results.

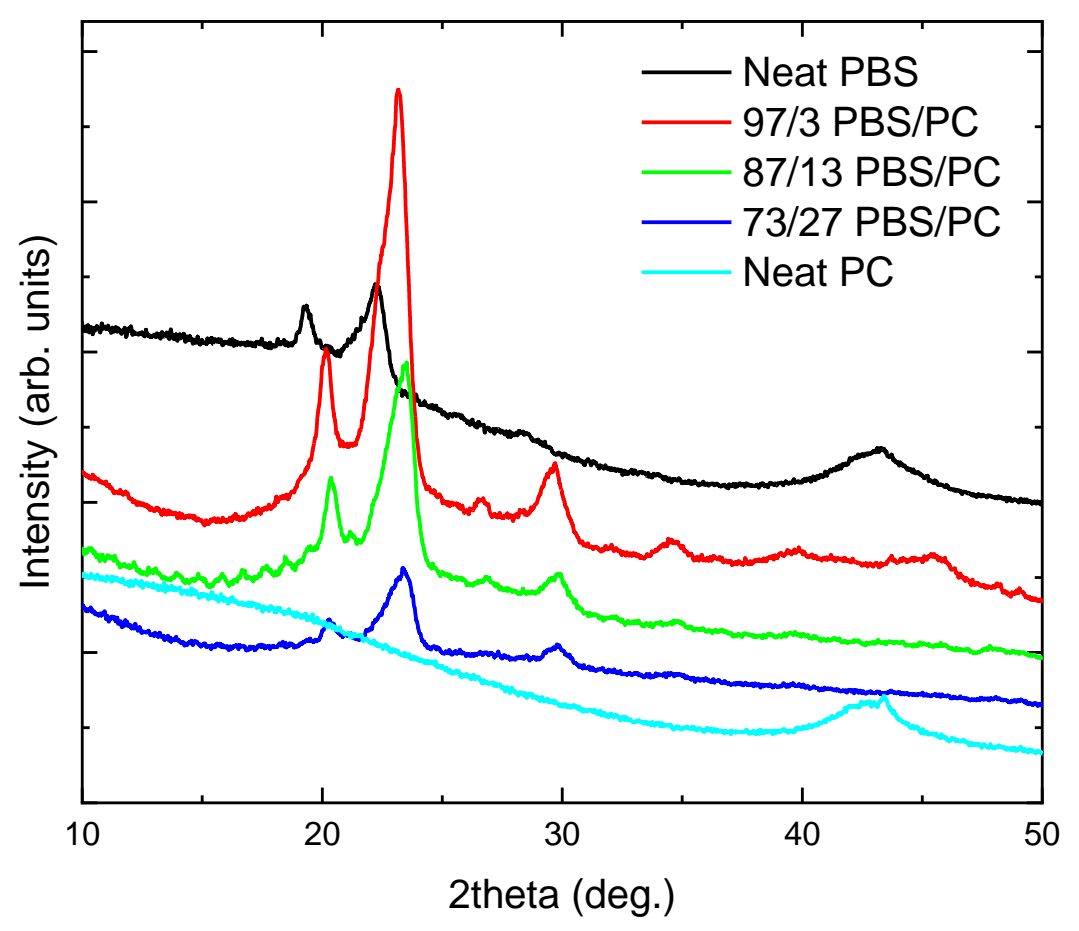

Figure 3 XRD graphs for neat PBS, neat PC, and the blends. 


\subsection{Thermal transitions: Differential Scanning Calorimetry (DSC)}

Figure 4 demonstrates the DSC (a) cooling, and (b) subsequent heating scans for all the investigated samples performed at $10{ }^{\circ} \mathrm{C} \mathrm{min}^{-1}$. According to the results presented in Figure 4(a-b), Neat PBS and the blends show a crystallization peak temperature $\left(T_{c}\right)$ between 69.5$71.9{ }^{\circ} \mathrm{C}$. In the subsequent heating scans, the melting temperature peak $\left(T_{m}\right)$ around 112.5$113.9^{\circ} \mathrm{C}$ is preceded by a cold crystallization peak $\left(T_{c c}\right)$ at approximately $95{ }^{\circ} \mathrm{C}$. This peak has been previously ascribed to the recrystallization of partially melted thin lamellae of PBS [35]. Previous reports on neat PBS showed a crystallization temperature of $76.3{ }^{\circ} \mathrm{C}$, a sharp exothermal peak temperature of $92{ }^{\circ} \mathrm{C}$ and a large endotherm at $114{ }^{\circ} \mathrm{C}$ [36]. Neat PC has a glass transition temperature value of $145.4{ }^{\circ} \mathrm{C}$. To have a clear picture of the results presented in Figure 4, the crystallization temperature $\left(T_{c}\right)$ and melting temperature $\left(T_{m}\right)$ values were plotted as a function of PC content in Figure 5.

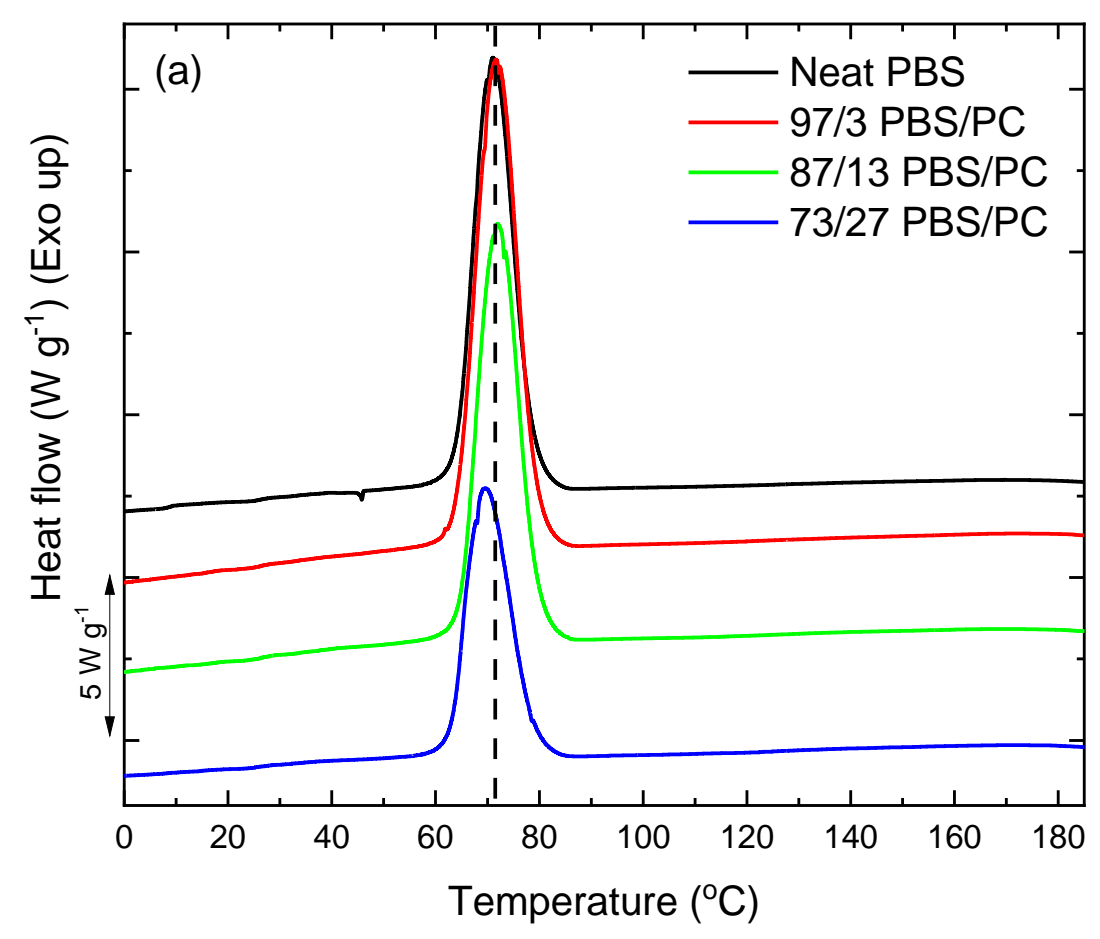




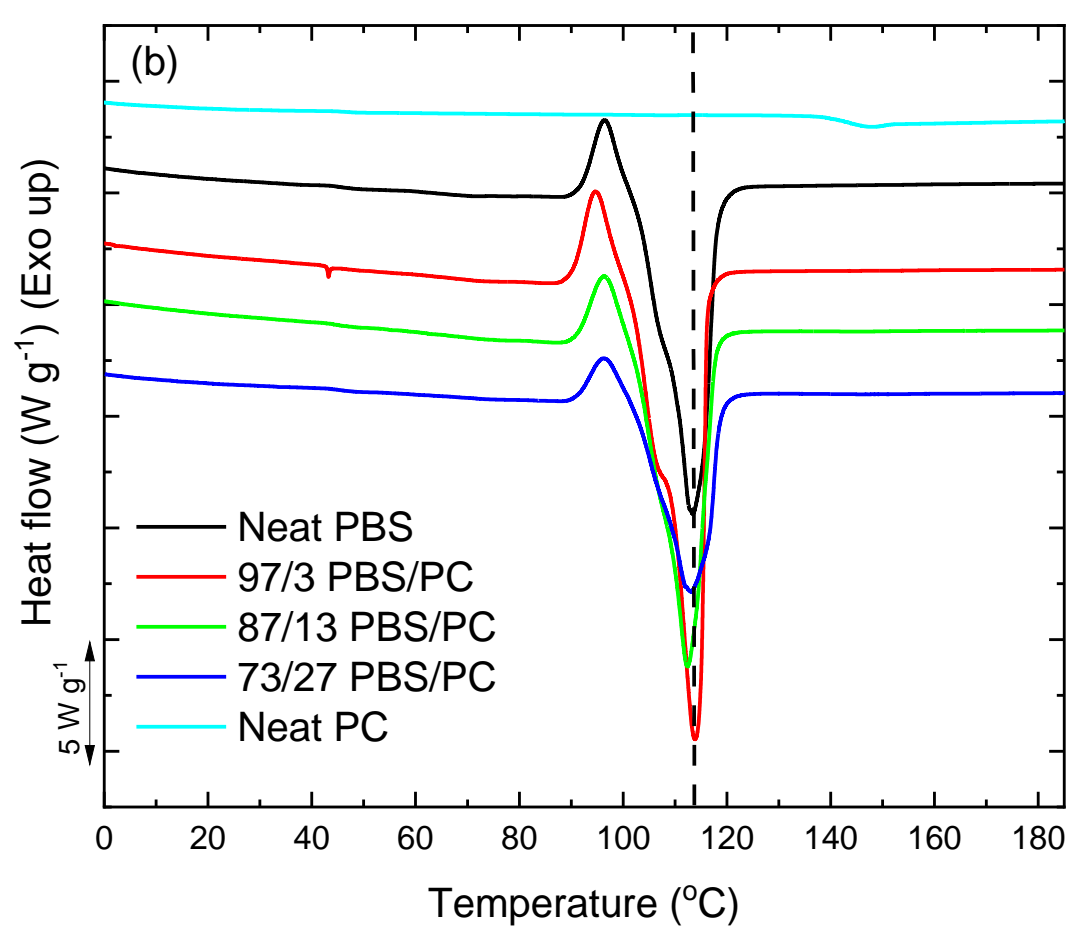

Figure 4 DSC (a) cooling and (b) second heating curves for neat polymers and the blends.

The $T_{c}$ of the PBS in the blends increase up to $13 \mathrm{wt} . \%$ PC. The increase indicates a nucleation effect of the PC that penetrated the PBS due to the partial miscibility in the blends. Above this content, the effect is not evident. There is a decrease in the nucleation effect as indicated by the drop in $T_{c}$ value. The decrease is probably related to the aggregation of PC that limits chain diffusion into the PBS phase as well as participation in nucleating PBS. The $T_{m}$ values of the PBS in the blends are lower than that of neat PBS up to $13 \mathrm{wt} . \%$ PC. This melting temperature depression is caused by the nucleation changes. Above this content, the $T_{m}$ value is higher than that of neat PBS and the other blends. This is because at this content, both effects are lost (i.e., nucleation and miscibility). 


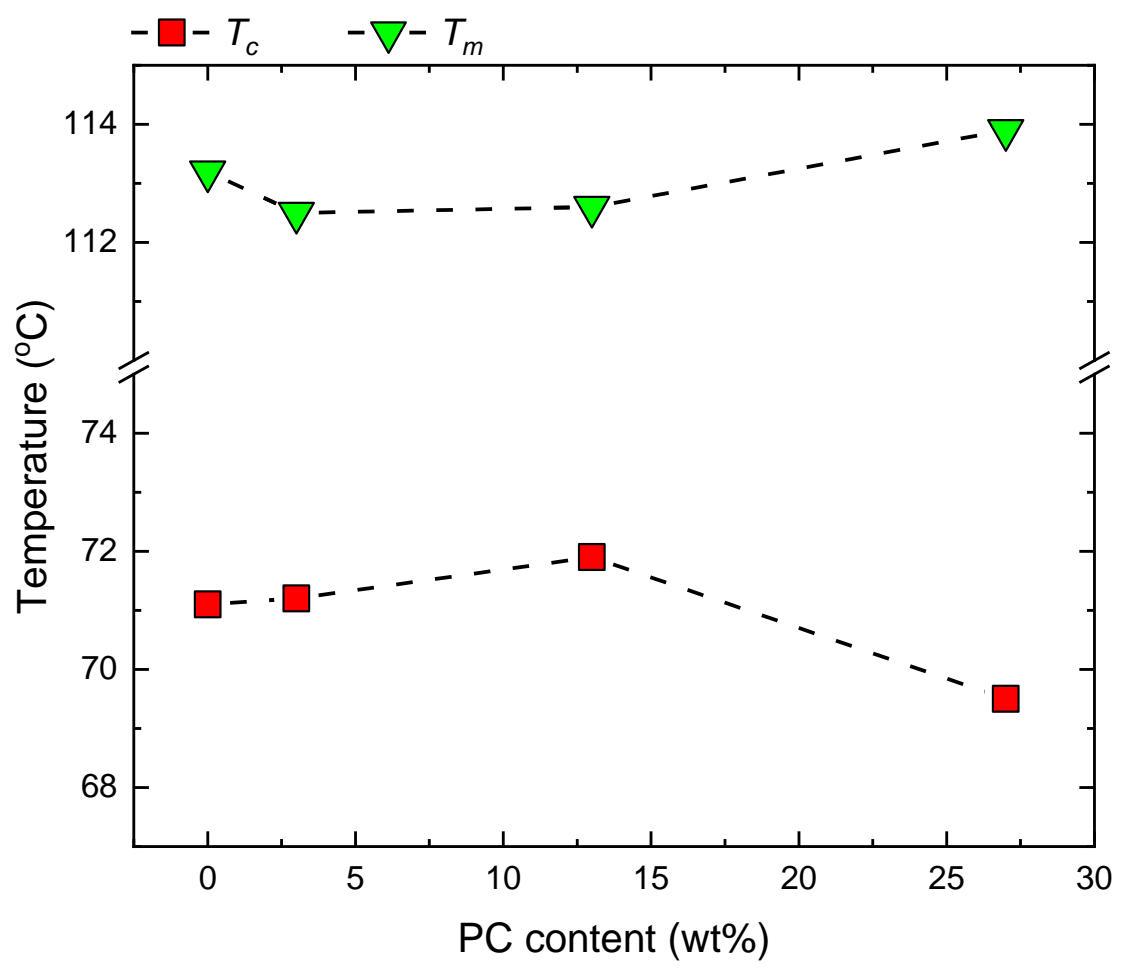

Figure 5 DSC cooling and second heating melting temperatures for neat PBS and the PBS/PC blends.

The normalised crystallization and melting enthalpy values shown in Table 2 and drawn in Figure 6 were determined according to Equations 1 and 2

$$
\begin{aligned}
& \Delta H_{c}{ }^{n}=\Delta H_{c} w \\
& \Delta H_{m}{ }^{n}=\Delta H_{m} / w
\end{aligned}
$$

where $\Delta H_{c}{ }^{n}$ and $\Delta H_{m}{ }^{n}$ are the crystallization and melting enthalpies normalised to the amount of the pure components in the sample, $\Delta H_{c}$ and $\Delta H_{m}$ are the crystallization and melting enthalpies of the pure components, and $w$ is the weight fraction of the pure components in the blend. If the PBS crystallized as a separate phase in the blend, one would expect the normalized melting enthalpies of the PBS in the blend to be the same as the melting enthalpy of neat PBS. In this case, there is an increase in crystallinity at low PC content (i.e., 3 wt.\%). This is because at low content of PC, there are two contributing effects (miscibility and nucleation). The individual PC chains may have been trapped in the amorphous phase of PBS and the nucleation 
effect may have promoted crystallization, thus leading to higher crystallinity. The nucleation effect is the highest at intermediate PC content (i.e., 13wt.\%), however, this might not suggest optimum miscibility. Elsewhere in literature [37], carbon black was fairly dispersed in a polyolefin matrix and gave rise to low melting enthalpy values, in contrast, zinc metal particles formed agglomerates in the same matrix and showed a high nucleation efficiency which led to a high crystallinity index as compared to carbon black. This is a clear indication that the nucleation ability of a dispersed phase should not be mistaken for its miscibility with a continuous phase. At high PC contents, the normalized melting enthalpies of the PBS in the blends are lower than of neat PBS. This is attributed to the loss of diffusion and limited phase mixing between the components in the blends. A similar trend is observed for the normalised crystallization enthalpy values.

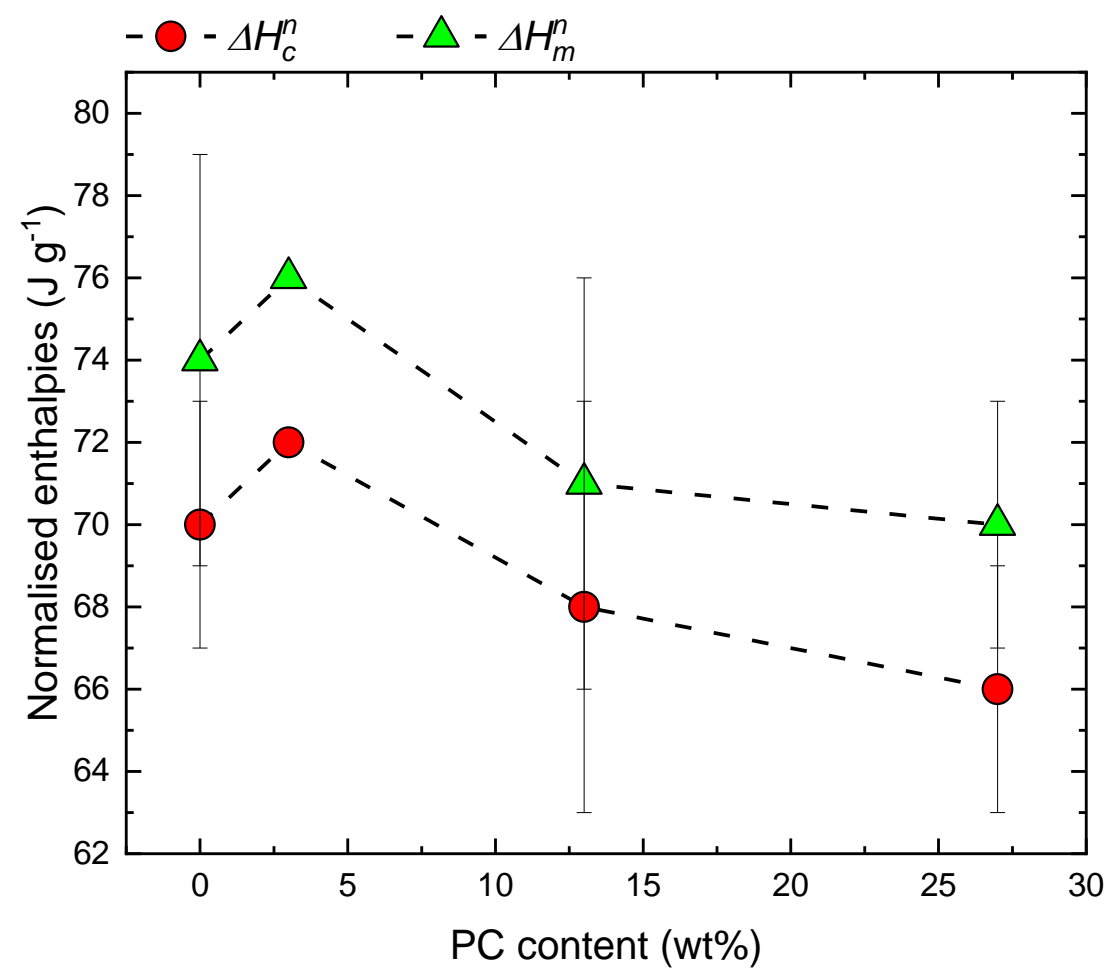

Figure 6 DSC normalized crystallization and second heating melting enthalpies for neat polymers and the blends. 
Table 2

\begin{tabular}{|c|c|c|c|c|c|c|c|c|c|}
\hline Sample & $T_{c}\left({ }^{\circ} \mathrm{C}\right)$ & $T_{m}\left({ }^{\circ} \mathrm{C}\right)$ & $T_{c c}\left({ }^{\circ} \mathrm{C}\right)$ & $\Delta H_{c}\left(\mathrm{~J} \mathrm{~g}^{-1}\right)$ & $\Delta H_{c}^{n}\left(\mathrm{~J} \mathrm{~g}^{-1}\right)$ & $\Delta H_{m}\left(J^{-1} g^{-1}\right)$ & $\Delta H^{n}{ }_{m}\left(\mathrm{~J} \mathrm{~g}^{-1}\right)$ & $\Delta \boldsymbol{H}_{c c}\left(\mathbf{J ~ g}^{-1}\right)$ & $T_{g}\left({ }^{\circ} \mathrm{C}\right)$ \\
\hline Neat PBS & $71.1 \pm 0.0$ & $113.2 \pm 0.3$ & $96.3 \pm 0.0$ & $70 \pm 3$ & $70 \pm 3$ & $74 \pm 5$ & $74 \pm 5$ & $9 \pm 1$ & - \\
\hline 97/3 PBS/PC & $71.2 \pm 0.1$ & $112.5 \pm 0.2$ & $95.0 \pm 0.1$ & $70 \pm 0$ & $72 \pm 0$ & $74 \pm 0$ & $76 \pm 0$ & $10 \pm 1$ & - \\
\hline 87/13 PBS/PC & $71.9 \pm 0.1$ & $112.6 \pm 0.1$ & $94.0 \pm 3.3$ & $59 \pm 5$ & $68 \pm 5$ & $62 \pm 5$ & $71 \pm 5$ & $8 \pm 0$ & - \\
\hline 73/27 PBS/PC & $69.5 \pm 0.4$ & $113.9 \pm 1.1$ & $96.5 \pm 0.6$ & $48 \pm 3$ & $66 \pm 3$ & $51 \pm 3$ & $70 \pm 3$ & $6 \pm 0$ & - \\
\hline Neat PC & - & - & - & - & - & - & - & - & $145.4 \pm 0.5$ \\
\hline
\end{tabular}




\subsection{Thermal degradation: Thermogravimetric analysis (TGA)}

Figures 7(a-b) shows TGA curves for the investigated samples. All the samples show a single degradation step with neat PBS decomposing at $394.6{ }^{\circ} \mathrm{C}$, and neat bisphenol A polycarbonate decomposing around $520.2{ }^{\circ} \mathrm{C}$. As expected, PC degrades at a much higher temperature, because of the presence of benzene rings and quaternary carbon atoms which form bulky and stiff chains that enhance rigidity and therefore, the thermal stability [38]. The absence of these structures in PBS means its aliphatic chains are more prone to thermal degradation, hence the lower thermal stability compared to PC.

In the case of the blends, the thermal stability did not seem to improve in comparison with neat PBS, despite the presence of a more thermally stable component (PC). This can be explained by the poor interfacial interaction between the two polymers, which formed blends with microstructures that are not uniform, especially at high PC contents. However, at low PC content ( $3 \mathrm{wt} . \%)$, there is a slight decrease in the thermal stability of the blend. This can be ascribed to the miscibility effect between the two component phases.

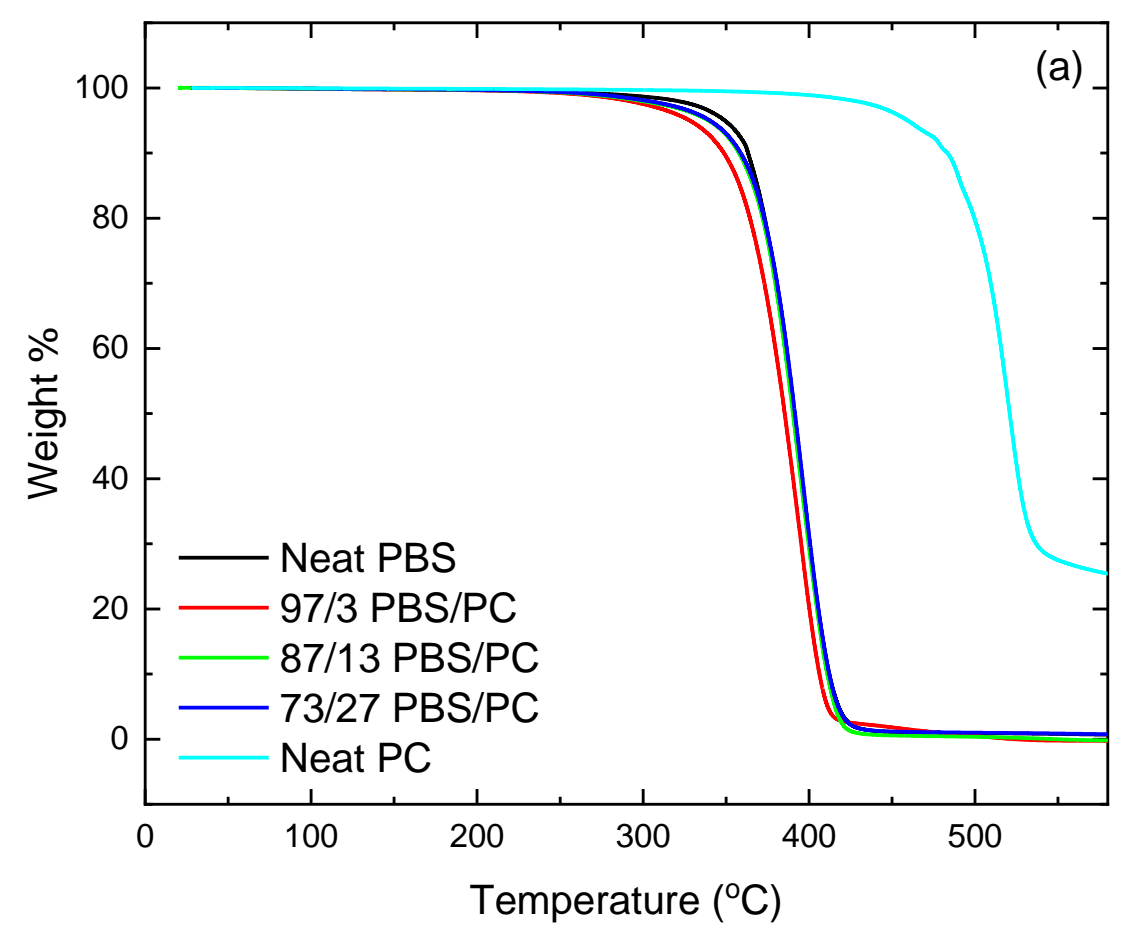




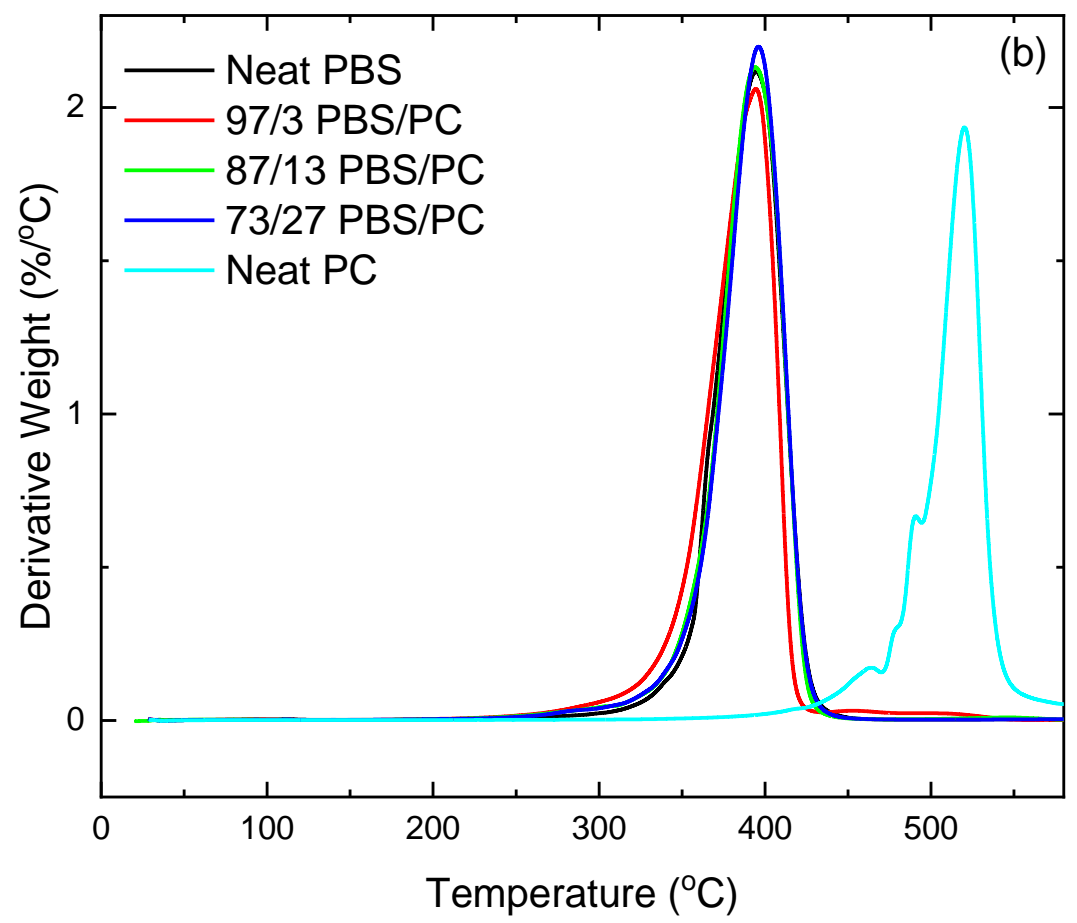

Figure $7 \quad$ TGA (a) thermal degradation and (b) derivative curves for neat polymers and the PBS/PC blends.

\subsection{Thermo-mechanical properties: Dynamic mechanical analysis (DMA)}

To access the viscoelastic behavior of the blends as a function of temperature, dynamic mechanical analysis was carried out. To investigate the possible reinforcement of PC on PBS, as well as the extent of miscibility in the amorphous phase, a composition dependence of the glass transition temperature $\left(T_{g}\right)$ was determined. Figure 8 a represents the transition from a glassy to rubbery state for all the investigated samples. At low PC content (i.e., 3 wt.\%), there is a marked improvement in the elastic modulus in comparison with the neat PBS and other blends. This may suggest a reinforcing effect of rigid PC on PBS due to the miscibility observed between the two polymers. Above this content, the blends do not show a specific trend over the whole temperature range of analysis. The observed elastic behaviour at high PC contents could be due to the presence of PC chains in the amorphous domains of PBS, rather than their reinforcing effect, largely due to the poor interfacial adhesion between components at these concentrations. It has been previously reported that the elastic moduli of composite materials are strongly dependent on the filler content rather than the interfacial adhesion between the 
reinforcing agent and the matrix [39]. In our case, PC acts as a particulate filler at low content (i.e., 3 wt.\%) and achieves a uniform dispersion due to better mixing with PBS, whereas aggregates dominate the blends at high PC contents and phase segregation is observed (as seen in the SEM images). Therefore, the homogeneous blend formed at low PC content leads to improved energy storage ability of the material, hence the high elastic modulus values.

The viscous moduli curves are presented in Figure $8 \mathrm{~b}$, and the shift in the $T_{g}$ to higher temperatures is evident in all the blends compared to neat PBS. According to literature [40], if two polymers are completely miscible, only one $T_{g}$ is observed, and its position is determined by the composition of the blend. For immiscible polymer blends, two distinct $T_{g}$ values are observed at the same temperatures as those of the individual components. However, when two polymers are partially miscible, two $T_{g}$ values are still observed, although they shift towards each other, with the extent of shifting being dependent on both the blend composition and the degree of miscibility. In our case, only the $T_{g}$ of the PBS component was recorded because the PBS melting temperature is well below the $T_{g}$ of PC. Moreover, as the PC content increases in the blends, the $T_{g}$ also increased, possibly indicating apparent interaction between the components. The intensity of $\tan \delta$ curves (results not included) decreases in all the blends compared to neat PBS, which further confirms the immobilization effect PC has on the amorphous chains of PBS. 

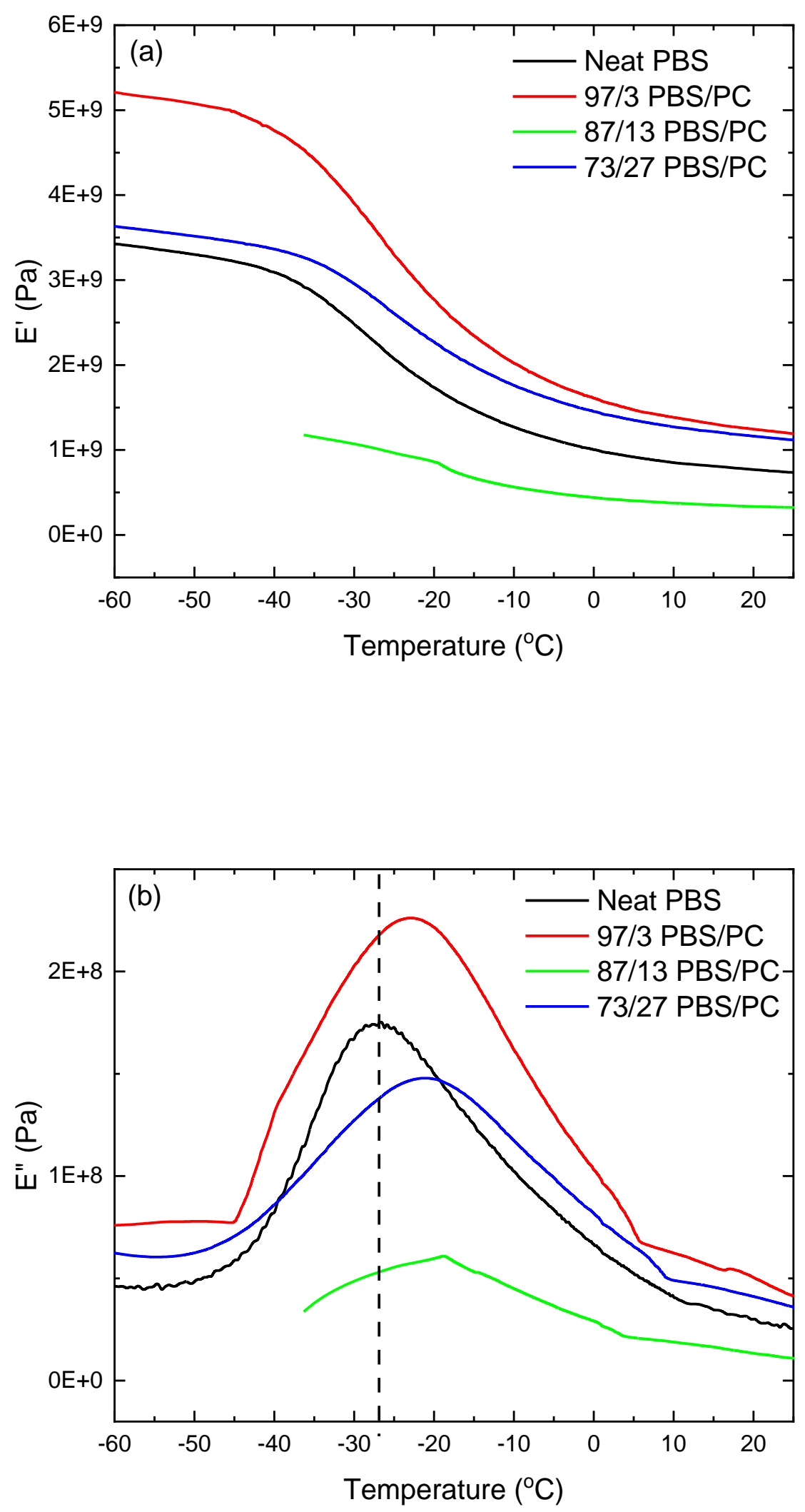
Figure 8 DMA (a) storge modulus (E'), and (b) loss modulus (E") curves for all the investigated samples.

\section{Conclusions}

In summary, PBS/PC blends of different weight\% ratios (97/3 PBS/PC, 87/13 PBS/PC and 73/27 PBS/PC) as well as neat PBS and neat PC were successfully prepared through melt extrusion. Chemical analysis using FTIR revealed an overlapping of the $\mathrm{C}-\mathrm{H}$ and carbonyl groups and also the development of double peaks at $1300 \mathrm{~cm}^{-1}$, indicating some chemical interaction between PBS and PC. Surface morphology analysis revealed a miscible polymer blend for the blend containing low content of PC (3 wt.\%), whereas phase segregation was observed with increasing PC content in the blends. The structural properties of the samples demonstrated that addition of PC to PBS improves crystallinity, with the blend having PC content of $3 \mathrm{wt} . \%$ showing high crystallinity in comparison to the rest of the samples and this observation was confirmed also by DSC normalized crystallization enthalpy values. DSC studies further revealed an increase in $T_{c}$ in the blends up to PC content of $13 \mathrm{wt} \%$, suggesting a nucleation effect of the PC that penetrated the PBS due to the partial miscibility in the blends. However, at a higher PC content (27 wt.\%); the $T_{c}$ declines; indicating a decrease in nucleation effect which is attributed to the aggregation of the chain diffusion into the PBS phase and participation in nucleating PBS. Furthermore, $T_{m}$ values of the PBS/PC blends up to 13 wt.\% were found to be lower than neat PBS indicating nucleation changes, whereas at PC content of 27 wt.\%, $T_{m}$ was higher than all the samples which indicated that both nucleation and miscibility effects are lost at this PC level.

Thermal degradation studies showed that PBS and the blends reach thermal stability at lower temperatures compared to PC, this is because PC contains benzene rings and quaternary carbon atoms that forms bulky and stiff chains, thus promoting rigidity, leading to higher thermal stability. As much as PC is present in all the blends; their thermally stability remained low, indicating poor interfacial interaction between PBS and PC, with the 3 wt.\% blend even showing a slightly lower thermal stability which is attributed to the miscibility effect between the PBS and PC phases.

Thermo-mechanical analysis indicated that the elastic modulus of the blend with PC content of $3 \mathrm{wt} . \%$ was improved in comparison to the rest of the samples and this is because at $3 \mathrm{wt} . \%$, PC takes a role of a particulate filler, leading to a uniform dispersion when mixed with PBS, whereas higher PC content results in formation of aggregates and phase segregation as 
confirmed by SEM analysis. Furthermore, the $T_{g}$ increased with increasing PC content, indicating interaction between the PBS and PC. The intensity of $\tan \delta$ declined in all the blends in comparison to the neat PBS, thus confirming immobilizing effect of PC on the amorphous chains of PBS.

With all these observations made, it is clear that to obtain the composition of the blend with preferred or required functional properties, the content/ratio of the neat components plays a crucial role, and these have to be controlled for more effective functional properties.

Acknowledgements: T.P. Gumede gratefully acknowledges the National Research Foundation for Black Academics Advancement Programme (BAAP) Grant Number: 129433 for financial support of the project.

Authors Contribution: Thandi Gumede conceived and designed the experiments. The X-ray diffraction experiments were performed by Katekani Shingange. The SEM measurements were performed by Pontsho Mbule. Bennie Motloung performed and analysed FTIR and DMA results. The writing of the manuscript was done mostly by Thandi Gumede, however, the rest of the authors also contributed by writing the parts related to the experiments they performed.

Conflict of Interest Statement: The authors declare no conflict of interest.

\section{References}

[1] J. H. Song, R. J. Murphy, R. Narayan, G. B. H. Davies, Philos. Trans. R. Soc. B 2009, $364,2127$.

[2] T. S. Mdletshe, S. B. Mishra, A. K. Mishra, J. Appl. Polym. Sci. 2015, 132, 42145.

[3] A. Bhatia, R. K. Gupta, S. N. Bhattacharya, H. J. Choi, Korea Aust. Rheol. J. 2007, 19, 125 .

[4] M. Gigli, A. Negroni, G. Zanaroli, N. Lotti, F. Fava, A. Munari, React. Funct. Polym. 2013, 73, 764 .

[5] A. Larrañaga, E. Lizundia, Eur. Polym. J. 2019.

[6] M. D. Sanchez-Garcia, E. Gimenez, J. M. Lagaron, J. Appl. Polym. Sci. 2008, 108, 2787.

[7] M. D. Sanchez-Garcia, A. Lopez-Rubio, J. M. Lagaron, Trends Food Sci. Technol. 2010, 21,528 .

[8] J. Li, Y. He, Y. Inoue, Polym. Int. 2003, 52, 949. 
[9] Y. Chen, L. Tan, L. Chen, Y. Yang, X. Wang, Braz. J. Chem. Eng. 2008, 25, 321.

[10] S. T. Sikhosana, T. P. Gumede, N. J. Malebo, A. O. Ogundeji, EXPRESS Polym. Lett. 2021, $15,568$.

[11] T. P. Gumede, A. S. Luyt, A. J. Müller, EXPRESS Polym. Lett. 2018, 12, 505.

[12] M. M. Reddy, A. K. Mohanty, M. Misra, Macromol. Mater. Eng. 2012, 297, 455.

[13] P. Nugroho, H. Mitomo, F. Yoshii, T. Kume, K. Nishimura, Macromol. Mater. Eng. 2001, 286, 316.

[14] Z. Qiu, M. Komura, T. Ikehara, T. Nishi, Polymer 2003, 44, 7749.

[15] E. Can, S. Bucak, E. Kinaci, A. C. Çalikoğlu, G. T. Köse, Polym. Plast. Technol. Eng. 2014, 53, 1178.

[16] Q. Liu, X. M. Zhou, J. Macromol. Sci. A J Macromol Sci A 2015, 52, 625.

[17] J. John, R. Mani, M. Bhattacharya. J. Polym. Sci. A Polym. Chem. 2002, 40, 2003.

[18] J. P. Mofokeng, A. S. Luyt, Polym. Test. 2015; 45, 93.

[19] J. P. Mofokeng, A. S. Luyt, J. Mater. Sci. 2015; 50, 3812.

[20] M. P. Motloung, V. Ojijo, J. Bandyopadhyay, S. S. Ray, J. Appl. Polym. Sci. 2019; 1.

[21] A. A. J. Ketelaars, Y. Papantoniou, K. Nakayama, J. Appl. Polym. Sci. 1997, 66, 921.

[22] Y. W. Cheung, R. S. Stein, B. Chu, G. Wu, Macromolecules 1994, 27, 3589.

[23] V. Balsamo, N. Calzadilla, G. Mora, A. J. Müller, J. Polym. Sci. B Polym. Phys. 2001, 39,771 .

[24] C. A. Cruz, D. R. Paul, J. W. Barlow, J. Appl. Polym. Sci. 1979, 23, 589.

[25] Y. S. Chun, J. Park, J. B. Sun, W. N. Kim, J. Polym. Sci. B Polym. Phys. 2000, 38, 2072.

[26] J. M. Jonza, R. S. Porter, Macromolecules 1986, 19, 1946.

[27] D. Herrera, J. C. Zamora, A. Bello, M. Grimau, E. Laredo, A. J. Müller, T. P. Lodge, Macromolecules 2005, 38, 5109.

[28] T. P. Gumede, A. S. Luyt, M. K. Hassan, R. A. Pérez-Camargo, A. Tercjak, A. J. Müller, Polymers 2017; 9, 709.

[29] T. P. Gumede, A. S. Luyt, R. A. Pérez-Camargo, A. Tercjak, A. J. Müller, Polymers 2018, $10,424$.

[30] A. K. Mohapatra, S. Mohanty, S. K. Nayak, J. Polym. Environ. 2014, 22, 398.

[31] S. F. Yao, X. T. Chen, H. M. Ye. J. Phys. Chem. B 2017, 121, 9476.

[32] C. Larosa, N. Patra, M. Salerno, L. Mikac, R.M. Meri, M. Ivanda, Beilstein J. Nanotechnol. 2017, 8, 2026.

[33] J. Dong, Y. Ozaki, Macromolecules 1997, 30, 1111.

[34] Y. Bu, J. Ma, J. Bei, S. Wang, Front. Bioeng. Biotechnol. 2019; 7, 1. 
[35] G. Liu, L. Zheng, X. Zhang, C. Li, S. Jiang, D. Wang, Macromolecules 2012, 45, 5487.

[36] S. A. Rafiqah, A. Khalina, A. S. Harmaen, I. A. Tawakkal, K. Zaman, M. Asim, M. N. Nurrazi, C. H. Lee, Polymers 2021, 13, 1436.

[37] B. T. Motloung, D. Dudić, J. P. Mofokeng, A. S. Luyt, J. Polym. Res. 2017, 24, 1.

[38] T. Artham, M. Doble, Macromol. Biosci. 2007, 8, 14.

[39] Z. Lule, J. Kim, Polymers 2019, 11, 1.

[40] J. D. Menczel, R. B. Prime, Thermal Analysis of Polymers: Fundamentals and Applications, John Wiley \& Sons, Hoboken, NJ 2009. 